\title{
Designing User Interfaces for Different User Groups: A Three-way Teleconference System for Doctors, Patients and Assistants Using a Remote Medical Robot
}

\author{
Gerald Stollnberger ${ }^{1}$, Manuel Giuliani ${ }^{1}$, Nicole Mirnig ${ }^{1}$, Manfred Tscheligi ${ }^{1}$, Krzysztof Arent $^{2}$, Bogdan \\ Kreczmer $^{2}$, Filip Grzeszczak ${ }^{2}$, Dorota Szczesniak-Stanczyk ${ }^{3}$, Radoslaw Zarczuk ${ }^{3}$, and Andrzej Wysokinski ${ }^{3}$
}

\begin{abstract}
We present the design for a three-way medical teleconference system for communication between a doctor, a patient, and an assistant. The system includes individual doctorpatient and doctor-assistant communication channels, as well as the capability of starting and stopping communication channels separately. The initial system design is based on results of a user requirement analysis. To evaluate the design, we conducted two user studies in which doctors, assistants, and patients used our teleconference system in a simulated examination scenario. The study results show that the general usability of our system was rated as good. However, doctors, patients, and assistants reported that they would like to receive better visualisation of the connection status for the communication channels and the system status of the robot in general. Based on these results, we present an updated design for the teleconference system. We also provide best practices which can help designers of medical teleconference systems.
\end{abstract}

\section{INTRODUCTION}

The shortage of medical personnel is a growing problem worldwide, even in developed countries. The World Health Organization (WHO) reports in [1] that Europe already has the need for more qualified doctors and the demand for doctors will steadily increase over the next years. Also at nighttime or in rural areas, the availability of medical specialists is a problem. One solution to overcome this challenge is to begin using teleconference systems to remotely provide consultancy for patients, or to ask fellow doctors for advice, who are experts in a given medical field. Doctors already apply this solution, e.g., to decide whether a stroke patient should come into the hospital for treatment.

In the ReMeDi project (Remote Medical Diagnostician ${ }^{1}$ ), we advance the remote examination of patients. The project goal is to implement a medical robot that enables doctors to interview and examine patients over remote distances. The ReMeDi robot supports a full examination of the patient, including an initial interview of the patient, two forms of examination (palpation and ultrasonography), and diagnosis reporting. The robot consists of two parts; one is stationed

\footnotetext{
*This work was supported by the $7^{t h}$ Framework Program of the European Union, contract number $\mathrm{n}^{\circ} 610902$.

${ }^{1}$ is with the Center for Human-Computer Interaction, Department of Computer Sciences, University of Salzburg, Austria, firstname. lastname@sbg.ac.at

2 is with the Chair of Cybernetics and Robotics, Wroclaw University of Technology, Poland firstname. lastname@pwr.edu.pl

3 is with the Department of Cardiology, Medical University of Lublin, Poland firstnamelastnamedumlub.pl

${ }^{1}$ http://www.remedi-project.eu
}

at the site of the doctor, the other part is located at the site of the patient (Fig. 1).

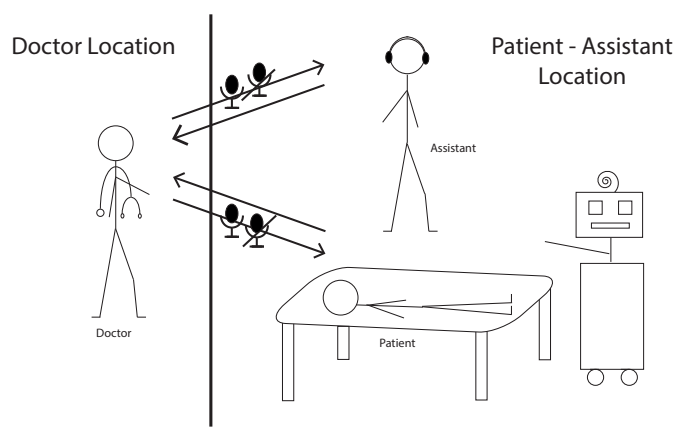

Fig. 1. The doctor location is depicted on the left side, patient and assistant on the right. Communication is symbolised by the arrows. Audio communication from doctor to patient can be muted by doctors.

The doctor controls the robot using a set of input devices. On the patient site (Fig. 2), an assistant monitors the examination and ensures the safety of the patient. The robot parts communicate over remote distances via the internet. More details on the robot are published in [2].

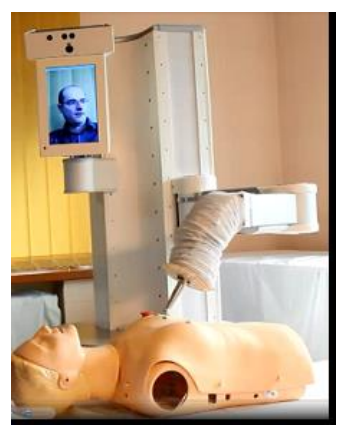

Fig. 2. The ReMeDi system on the site of the patient which shows the robot with an attached screen to display the doctor's face.

A central component of ReMeDi is a three-way teleconference system enabling a continuous communication between doctor, patient, and assistant. In this work, we focus on the question of how this teleconference system needs to be designed to meet the needs for all three user groups (distant doctors examining patients, patients getting examined, and assistants supporting the procedure at the patients location).

We first describe the results of a user requirements analysis 
that we conducted as part of the implementation plan of the ReMeDi robot (Section III). One of the essential requirements that was mentioned by all user groups is the availability of a secure and robust teleconference system. Second, we give an overview of the initial user interface design of the system (Section IV). Third, we show the results of two evaluations of the teleconference system (Section V). In two user studies, we asked doctors and assistants to use the system in a simulated examination scenario. This allowed us to assess the overall usability of the system and gain feedback on the user interface design by the targeted end users. Based on the results from these evaluations, we update the design of the user interface (Section VI).

\section{BACKGROUND}

Most teleconference solutions in medical care apply commonly available stand-alone web-based programs for communication rather than those integrated in larger medical systems. It is quite common for doctors to seek advice from experienced colleagues via email, text chats, and video conferences. This is especially true in remote areas which are lacking medical specialists, but also in big medical centres when a consultation is needed for immediate decisionmaking. Researchers study this professional-to-professional remote contact in such areas as expert-guided surgeries [3], orthopaedics [4], endoscopic procedures [5], dermatology [6], radiology, and shipboard medicine [7].

Regarding doctor-patient interaction, potential applications are already under investigation. Advanced research is beeing conducted on tele-psychotherapy. Jenkins et al. [8] analysed the results of remote psychotherapy compared to in-person psychotherapy. The results show comparable treatment satisfaction and similar ratings of therapeutic alliance. Shenai et al. [9] created and tested virtual interactive presence systems for real-time long-distance surgical collaboration. Their aim was to visually and verbally interact with doctors while they identify anatomical structures. They guided surgical manoeuvres and discussed strategies, as well as mentored less experienced personnel located at the surgical site by remotely located experts. Other possible fields under investigation are psychiatry [10], neurology (assessing stroke scale) [11], and rehabilitation [12] as well as how to control robotic telepresence platforms from the users' perspective [13].

Studies show that teleconferencing is not inferior to faceto-face consultation, for example, in recognizing abnormal skin changes [14]. There are also fully developed medical systems that use teleconference elements to submit patient information remotely. For example, Bauer et al. [15] presented a system for fully remote robot-assisted surgery that uses a teleconference system to send video to the doctor for remote needle placement. In the ReMeDi project, we aim for a three-way teleconference system in a larger medical system capable of performing physical examination and ultrasonography. The tele-conference system should meet the needs of doctors, patients, and assistants equally. There is no robotic medical system which enables doctors to conduct a complete physical examination including tele-conferencing, palpation and ultrasonography so far. Our system could allow doctors to remotely conduct a complete physical examination or ultrasonography allowing patients in regions with lower medical availability a proper diagnosis.

\section{USER REQUIREMENTS}

As the first step for the implementation of the ReMeDi robot, we conducted a user requirement analysis. The goal was to understand the needs of the involved user groups. We determined the preferences of the users in robot appearance, handling, and the required technical features. For this, we conducted two focus groups with 9 doctors and assistants and two focus groups with 9 patients in total. We carried out the interviews in Poland and Austria. For full details on the process of interviewing the focus groups, please refer to [16] and [17].

One main outcome of the user requirements analysis was that all three user groups regard the continuous communication between doctor, assistant, and patient as an essential feature for a correct diagnosis. In the following list, we show a partial excerpt of the full list of user requirements. We only list requirements that are directly or indirectly related to communication. Each requirement is marked with a letter and a number. The letter represent whether a requirement was raised by the Doctors, the Assistants, or the Patients.

D1 Doctors want to communicate with the patient during the whole examination using video and audio channels.

D2 Doctors want to have an additional communication channel to the assistant that is separated from communication with the patient and can be put into a private mode in which the patient cannot observe the doctorassistant communication.

D3 Doctors would like to observe local changes of the patient's body temperature and assess moisture of a patient's skin.

D4 Olfactory information can be crucial to make a diagnosis in some cases, for example, if the patient has been drinking alcohol, has vomited, or has diarrhoea.

D5 Doctors want to have a secure data connection between the two modules of the ReMeDi system.

D6 Doctors would like to have a real-time interaction with the patient, without disturbing delays.

P1 Patients want to communicate with the doctor during the whole examination process, verbally and visually, preferably keeping eye contact. The patients reported they fear that the social connection with the doctor could suffer if the doctor is not looking at them.

P2 Patients are strictly against a private communication channel between doctor and assistant.

P3 Patients want to have a secure data connection between the two modules of the ReMeDi system.

A1 Assistants should be able to transfer additional data to doctors, for example, patient temperature, blood pressure, smell, and skin wetness. 
Four of the user requirements are directly related to the communication between doctor and patient or doctor and assistant (D1, D2, D6, P1). Three of the requirements are related to submitting additional information about the patient's condition to the doctor (D3, D4, A1). Doctors and patients voiced concerns about their privacy and the security of the patient's data (D5, P2, P3). It is interesting to note that requirements D2 and $\mathbf{P 2}$ are contradictory to each other. Doctors want to have a private communication channel to the assistant, but patients do want to hear the communication between doctor and assistant at all times. The private transfer of information between doctor and assistant is necessary, e.g., for diseases in which patients show symptoms that are similar to human behaviour under the influence of alcohol or drugs. In such a case, only the assistant, who can directly observe the patient, is able to properly evaluate the state of the patient (e.g., by the smell of the patient).

In the following section, we present the design of the graphical user interface of the teleconference system. We will also discuss how the implementation is based on the collected user requirements and how we decided to resolve the contradiction in requirements $\mathbf{D 2}$ and $\mathbf{P 2}$.

\section{GRAPHICAL USER INTERFACE}

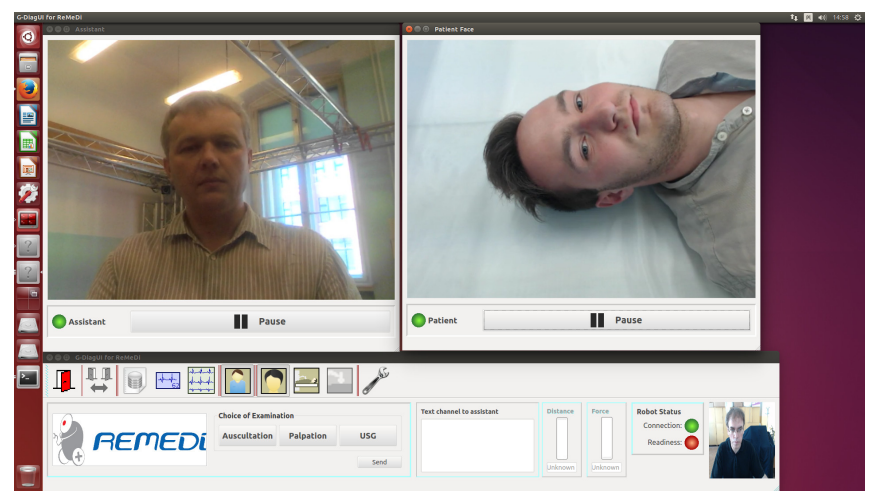

Fig. 3. Graphical user interface for the doctor. It contains two windows for indivdiual communication channels to assistant (left) and patient (right). The third window in the lower part is for displaying additional information and for setting preferences.

Based on the user requirements, we designed an initial graphical user interface (GUI) controlled by mouse and keyboard for the doctor to communicate with assistant and patient. Fig. 3 shows a screenshot of the GUI. We implemented the software as a custom-built package using the libraries GStreamer-0.10 for video and audio streaming, QT4 for the graphical interface, and the ROS framework for peer to peer communication. It consists of three separate windows. The upper two windows contain video channels to the assistant and to the patient. In the third window, which we will refer to as data window, the doctor can access basic functionality of the robot. He/she can also customize system preferences, for example, screen brightness and sound volume of the communication channels.

Assistant and patient can both see the doctor and communicate with him/her. For the patient, the ReMeDi robot has an attached screen that shows the face of the doctor. Fig. 2 shows robot and screen on the patient's site. The assistant has an additional laptop with a communication window to the doctor, which is similar to the doctor-assistant communication window in Fig. 3. In the user requirements analysis, the patients specified that they did not want to have private communication between doctor and assistant. However, in order to provide the best possible diagnosis, a private doctor-assistant communication is sometimes necessary. Therefore, the doctor can interrupt both communication channels temporarily. This feature is symbolized by a pause button, as can be seen in Figure 3. The assistant is wearing wireless headphones to receive the voice of the doctor in private. For details on the technical implementation of the GUI, please refer to [18] and [19].

We conducted two studies with the initial user interface design, to get feedback from future users of the system.

\section{USER STUDIES}

We conducted two user studies to identify usability problems of the initial GUI design. In the first study, we asked doctors to conduct an interview with a patient using the teleconference system (section V-A). In the second study, we asked assistants to talk to a doctor using the system (section V-B). Both studies were approved by the ethics committee of the Medical University of Lublin. In this section, we present the details and results of these studies.

\section{A. Doctor-Patient Communication Study}

In the first study, we asked doctors to interview a patient. The goal of this study was to assess the usability of our initial teleconference GUI and to collect suggestions for improvements by the doctors.

1) Study Procedure: Before the study, the experimenters gave instructions to the doctor about the GUI and how to operate them. The doctors also got the information that the patient is an actor and that the patient will display predefined symptoms. The behaviour of the patient was spontaneous during the examination, but he displayed the same symptoms for each doctor. During the study, we placed doctor and patient in two separate rooms. They did not have any other communication channels beside the channels provided by our GUI. The different computers were connected by a wireless network. We told the doctors to interview the patient and to follow their regular routine for patient interviews.

2) Participants: In this study, 26 participants (13 female, 13 male) took part. The mean age of the participants was 30.08 ( $\mathrm{SD}=7.50$ ) years. We recruited all participants from the hospital staff at the Medical University of Lublin, Poland. The participants reported that they had been working as doctors for 5.30 years on average $(S D=5.77)$. Many junior doctors or medical students, who were still in training to become doctors, participated in our study.

3) Measurements: After the experiment, doctors filled in three questionnaires. The first questionnaire was the System Usability Scale (SUS) [20], a questionnaire that contains 10 items to assess the usability of user interfaces. The second 
questionnaire was the Attitude Towards Technology scale (ATT) [21], a questionnaire that contains 10 items (revised perceived ease of use) to assess the attitude of the participant towards technology. The third questionnaire was a subset of the Godspeed questionnaire (GS) [22], containing three items to assess the perceived safety of the participants. All of these questionnaires are standardized and have been used in other user studies before. SUS and ATT use five-point Likertscales for the ratings, whereas the Godspeed questionnaire is based on differential semantic scales. Additionally, we prepared a questionnaire in which we asked the participants for improvement suggestions with open questions and their demographic data.

4) Results: The participants rated the usability of our teleconference system with an average of $78.30(\mathrm{SD}=15.57)$ points on the SUS. SUS ranges from 0 to 100 points. According to [23], this corresponds to be between a "good" and "excellent" usability. The participants rated their own attitude towards technology with $2.38(\mathrm{SD}=1.06)$ points on the ATT. The ATT Likert scale ranges from 1 (positive) to 5 (negative attitude). The participants rated their perceived safety while using the system with 3.15 (slightly negative, SD $=0.89$ ) points on the GS. We inspected the internal reliability of all questionnaires by calculating Cronbach's alpha to ensure the consistency of results across the questionnaire items. For the ten SUS items, Cronbach's alpha was 0.89 (good reliability); for the ten ATT items, Cronbach's alpha was 0.96 (excellent reliability); and for the three GS items, Cronbach's alpha was 0.70 (acceptable reliability).

In the open part of the questionnaire, the participants gave many positive comments. They especially perceived the main feature of our teleconference system, the remote consultation of patients with doctors, as useful. They also mentioned that the two separate communication channels to assistant and patient are convenient. The doctors had some suggestions for improving the system. They often mentioned that the video and sound quality of the system should be improved. Only one participant mentioned that the communication delay caused by the transmission speed was too long. A few participants had concrete suggestions for improving the interface design. They suggested that the system should display an alert to the patient, when the doctor pauses the communication channel or when there are connection issues with video or sound. Furthermore, they reported that the play/pause function in its initial implementation was confusing and that icons in the GUI should be accompanied by additional text. Finally, many participants requested that they want to be able to move the camera view of the patient camera. This was not part of the study we present here, but it will be a feature in the final ReMeDi robot.

\section{B. Assistant-Doctor Communication Study}

In the second study, we asked assistants to communicate with a doctor using our teleconference system. The goal of this study was to assess the usability of the teleconference GUI and the functionality to submit additional information about the patient and the robot status.
1) Procedure: Before the study, the experimenters gave instructions to the assistants about the GUI and how to submit additional information to the doctor. The assistants also received the information that the doctor is an actor. During the study, we placed assistant and doctor in two separate rooms. They did not have any other communication channels beside the channels provided by our GUI. The doctor first asked the assistant to provide a short report on the preparations for starting an examination with the ReMeDi robot. For this, the assistant had to submit all necessary information to the doctor using our teleconference system that was installed on a separate laptop for the assistant. Additionally, the doctor used the private communication channel to the assistant to give further instructions.

2) Participants: In this study, 7 participants (6 female, 1 male) took part. The mean age was $30.86(\mathrm{SD}=8.76)$ years. We recruited all participants from the hospital staff at the Medical University of Lublin. They were not doctors, but worked as medical staff at the hospital, for example, as a nurse. We asked the participants for their highest degree of education; 4 had a graduate degree, 1 participant had an apprenticeship, 1 participant finished high school, and 1 participant had no higher education degree.

3) Measurements: We used the same set of questionnaires that we described in Section V-A.3.

4) Results: First, we inspected the internal reliability of all questionnaires by calculating Cronbach's alpha. For the 10 SUS items, Cronbach's alpha was 0.87 (good reliability); for the 10 ATT items, Cronbach's alpha was 0.69 (acceptable reliability); and for the 3 GS items, Cronbach's alpha was 0.46 (bad reliability). We excluded the perceived safety from further analysis due to the bad reliability score. The assistants rated the usability of our system with an average of 67.50 $(\mathrm{SD}=15.68)$ points on the SUS. According to [23], this corresponds to be between an "ok" and "good" usability. The participants rated their own attitude towards technology with $2.01(\mathrm{SD}=0.40)$ points on the ATT.

In the open part of the questionnaire, the assistants also rated the ability to conduct remote consultations as very useful. Similar to the doctors, they suggested to improve the video and sound quality of the system, but they had no complains about the connection speed.

\section{Discussion}

From the user answers to the SUS from both studies combined, we interpret that the usability of our teleconference system is fairly good for an initial system version. The ATT scale was slightly positive but very close to neutral. The standard deviation in the answers to the ATT scale was quite high in the communication studies. This indicates that some of our participants (doctors and assistants) have a more positive attitute towards technology than others. Due to the fact, that we found no statistically significant correlations between ATT and SUS, we conclude, that our system was usable (good SUS score), no matter if users have a positive or negative ATT. 
The doctors rated the perceived safety as average. The GS asks the users about their emotional state while using the system. The fact that perceived safety was received just as average, indicates that the users are not completely confident to use the system. We cannot infer from the data if the unease of the users stems from concerns about the privacy of their communication or from another source. We plan to investigate this further with a questionnaire aiming especially in users' emotional states in the next evaluations of the ReMeDi robot. Some doctors reported that they would like to have a better video and audio quality in the teleconference system. We will improve this in the next version of the system. It is interesting to note that we only received one comment about the latency of the system. In the user requirement analysis, the doctors requested a real-time interaction with the patients without disturbing delays (D6). The qualitative study results suggest that we achieved this user requirement.

Many of the improvement suggestions by the study participants are related to displaying the system status. First, the users found the play/pause function to stop and start the communication channel confusing. Second, they also reported that they would like to see the connection status (e.g., connected, disconnected, paused, established connection) of the communication channel indicated in the GUI.

In the next section, we present the revision of the GUI for our system, which we made based on the study results.

\section{GUI IMPROVEMENTS}

Based on the results of the user studies, we implemented a second version of the GUI for our teleconference system now using GStreamer-1.0, which reduces the latency between the different hosts to hardly noticeable, and QT5. First, we changed the appearance of the pausing mechanism for the communication channels. In the initial version, we used a single button for pausing and resuming video and audio connection. The displayed function on the button switched between play and pause mode for each button press, which was confusing for the study participants. For the updated design, we followed two design principles: a button function cannot be changed, and button icons should be accompanied with additional text and a unique color if necessary to enhance their meaning and clarity. With these principles in place, we redesigned the play/pause function for the communication channels.

Additionally to the play/pause function, we revised the design of the rest of the GUI. In the second implementation, most interface elements use two output modes for representing information, namely text and a graphical icon.

Study participants reported that they would like to have an indicator that shows doctor and patient whether the communication channel is connected or not. In the initial version of our GUI, we used a LED-like indicator to indicate the connection state. There are three connection states; no connection if remote site is off, paused if there is no examination ongoing, or live. In the improved GUI, we enhanced this information by showing icons and text directly in the video display window. The background colour of the windows changes from grey to green when the system attempts to connect to assistant or patient. Fig. 4 shows an example for the connection status. In the communication window on the left, the system connects to the assistant. In the communication window on the right, the system indicates that there is no connection with the patient computer.

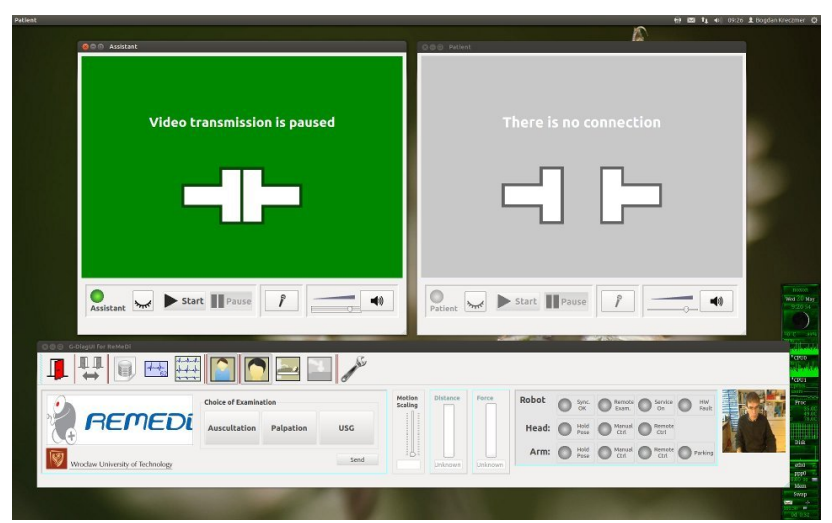

Fig. 4. Presentation of connection state in the assistant and patient communication channels.

We also changed the system behaviour for pausing and continuing a connection. Doctor and assistant both have the rights to pause and start their communication channel. Only the doctor can pause the communication channel between themself and the patient. We changed the communication mode for a private communication between doctor and assistant that cannot be heard by the patient. In this mode, the doctor can stop his/her own audio transmission to the patient, while the transmission from patient to doctor is still alive. The assistant should stay with patient and robot the whole time but they can listen to the doctor by cordless earphones. In an updated version, an assistant can also send critical information via text channel to the doctor. This allows doctors to discretely consult with the assistant while simultaneously observing the patient.

In order to ensure that the doctors are able to differentiate between normal and private communication mode, we introduced an indicator to represent this in the GUI. The indicator has the form of an eye. The eye is open when the system is switched to normal communication mode, and it is closed when the doctor switches to the private communication mode. To unify the interface of the communication channel for doctor and assistant, we introduced the same icon in the doctor and the assistant GUI. Fig. 5 shows an example of the GUI on the doctor site set to private mode.

\section{CONCLUSION}

We presented a teleconference system for doctors, patients and assistants using a medical robot. The initial design of the system was based on a user requirements analysis that we conducted with the involved user groups (doctors, assistants, and patients). We evaluated the system in two user studies. The study results show that the system has a good usability for all user groups. The study participants had improvement suggestions regarding the video and audio 


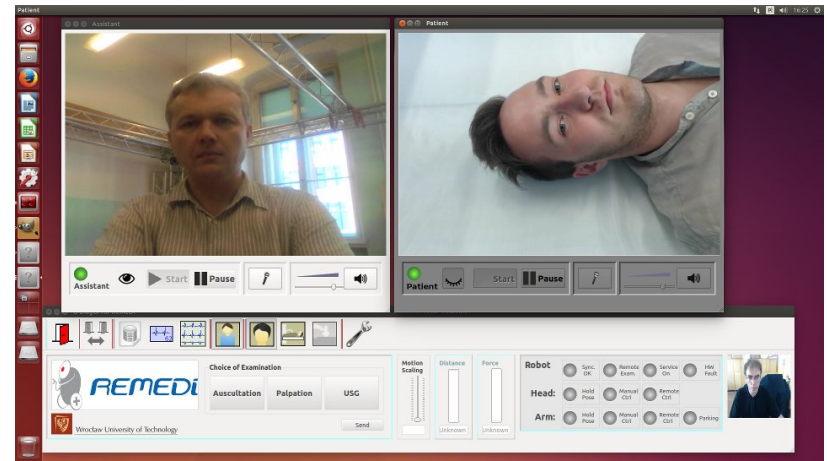

Fig. 5. Improved GUI from the doctor's perspective, using the private channel to the assistant (patient speaker is muted).

quality. Additionally, the users asked for a better indication of system status in the teleconference system. We will also propose a more consistent color coding such as yellow indicating transitional status, red for warning and green for "ok". Based on these suggestions, we updated the user interface. The repetitive user analysis early in the design process showed great promise, as at that time, the flexibility of the implementation phase is much higher than later in the project.

With implementation of the teleconference system and the user studies completed, we propose the following best practices for designers of teleconference systems in the medical context to allow a proper diagnosis:

- The system status needs to be visible for all involved users.

- System functionalities need to be consistently visualized by a combination of icons, colors, and if possible also by text.

- Colliding user requirements make compromises necessary. In such a case, the requirement which is more important to solve the task effectively should be preferred.

We will conduct a user study with patients, once the system is fully integrated, to further investigate in the patients' needs in the teleconference system also in relation with other robot functionalities with an additional analysis also on performance and error data. In future work, we will integrate our teleconference system into the existing ReMeDi robot prototype. After the integration, we will evaluate the whole system with healthy volunteers, assistants and doctors in a real examination scenario.

\section{REFERENCES}

[1] Actionforglobalhealth.eu. Addressing the global health workforce crisis: challenges for France, Germany, Italy, Spain and the UK. Technical report, World Health Organisation, January 2011. [Online; accessed 04-August-2015].

[2] A. Peer, M. Buss, B. Stanczyk, D. Szczesniak-Stanczyk, W. Brzozowski, A. Wysokinski, M. Tscheligi, C. A. Avizzano, E. Ruffaldi, L. van Gool, A. Fossati, K. Arent, J. Jakubiak, and M. Janiak. Towards a Remote Medical Diagnostician for Medical Examination. In Proceedings of the NextMed/MMVR21, 2014.

[3] S. Shimizu, HS. Han, K. Okamura, C. Bao, Y. Kitamura, N. Nakashima, and M. Tanaka. Live surgery and teleconferencing at the 19th World Congress of the International Association of Surgeons, Gastroenterologists and Oncologists (IASGO) in Beijing. Hepatogastroenterology, Sep-Oct(58):110-111, 2011.
[4] P. Aarnio, H. Lamminen, J. Lepisto, and A. Alho. A prospective study of teleconferencing for orthopaedic consultations. Journal of Telemedicine and Telecare, 5(1):62-66, 1999.

[5] S. Shimizu, S. Itaba, S. Yada, S. Takahata, N. Nakashima, K. Okamura, R. Rerknimitr, T. Akaraviputh, X. Lu, and M. Tanaka. Significance of telemedicine for video image transmission of endoscopic retrograde cholangiopancreatography and endoscopic ultrasonography procedures. Journal of hepato-biliary-pancreatic sciences, 18(3):366374, 2011.

[6] H. S. Pak, D. Harden, D. Cruess, M. Welch, R. Poropatich, National Capital Area Teledermatology Consortium, et al. Teledermatology: an intraobserver diagnostic correlation study, part I. Cutis-New York, 71(5):399-403, 2003

[7] P. H. Stoloff, F. E. Garcia, J. E. Thomason, and D. S. Shia. A cost-effectiveness analysis of shipboard telemedicine. Telemedicine Journal, 4(4):293-304, 1998.

[8] M. A. Jenkins-Guarnieri, L. D. Pruitt, D. D. Luxton, and K. Johnson. Patient Perceptions of Telemental Health: Systematic Review of Direct Comparisons to In-Person Psychotherapeutic Treatments. Telemedicine and e-Health, 2015.

[9] M. B. Shenai, R. S. Tubbs, B. L. Guthrie, and A. A. CohenGadol. Virtual interactive presence for real-time, long-distance surgical collaboration during complex microsurgical procedures. Journal of Neurosurgery, 121(2):277-284, 2014.

[10] K. Myers, J. Valentine, and S. Melzer. Child and adolescent telepsychiatry: utilization and satisfaction. Telemedicine and e-Health, 14(2):131-137, 2008

[11] B. M. Demaerschalk, S. Vegunta, B. B. Vargas, Q. Wu, D.D. Channer, and J.G. Hentz. Reliability of real-time video smartphone for assessing National Institutes of Health Stroke Scale scores in acute stroke patients. Stroke, 43(12):3271-7, 2012.

[12] W. Durfee, J. Carey, D. Nuckley, and J. Deng. Design and implementation of a home stroke telerehabilitation system. In Engineering in Medicine and Biology Society, 2009. EMBC 2009. Annual International Conference of the IEEE, pages 2422-2425, Sept 2009.

[13] S. Radmard, A.J. Moon, and E.A. Croft. Interface design and usability analysis for a robotic telepresence platform. In 24th IEEE International Symposium on Robot and Human Interactive Communication (RO-MAN), pages 511-516, Aug 2015.

[14] H. S. Pak. Teledermatology and teledermatopathology. Semin Cutan Med Surg., 21(3):179-89, 2002.

[15] J. Bauer, B. R. Lee, D. Stoianovici, J. T. Bishoff, S. Micali, F. Micali, and L. R. Kavoussi. Remote percutaneous renal access using a new automated telesurgical robotic system. Telemedicine Journal and EHealth, 7(4):341-346, 2001.

[16] G. Stollnberger, C. Moser, C. Zenz, M. Tscheligi, D. SzczesniakStanczyk, M. Janowski, W. Brzozowski, and A. Wysokinski. Capturing Expected User Experience of Robotic Systems in the Health Care Sector. In Proceedings of the Austrian Robotics Workshop, ARW '14, pages 42-46, 2014.

[17] G. Stollnberger, C. Moser, E. Beck, C. Zenz, M. Tscheligi, D. Szczesniak-Stanczyk, M. Janowski, W. Brzozowski, R. Blaszczyk, M. Mazur, and A. Wysokinski. Robotic Systems in Health Care. In Proceedings of the 7th International Conference on Human System Interaction, pages 276-281, 2014.

[18] K. Arent, M. Cholewinski, J. Jakubiak, and B. Kreczmer. Initial project of a graphical user interface for DiagUI. Technical Report 11, Wroclaw University of Technology, 2014.

[19] B. Kreczmer, F. Grzeszczak, D. Szczesniak-Stanczyk, K. Arent, and B. Stanczyk. Video conferencing applications for ReMeDi robotic system. Journal of Medical Imaging and Health Informatics, 5(8):16221630,2015

[20] J. Brooke. SUS-A quick and dirty usability scale. Usability evaluation in industry, 189(194):4-7, 1996.

[21] M. Y. Chuttur. Overview of the Technology Acceptance Model: Origins, Developments and Future Directions. Sprouts: Working Papers on Information Systems, 9(37), 2009.

[22] C. Bartneck, D. Kulić, E. Croft, and S. Zoghbi. Measurement instruments for the anthropomorphism, animacy, likeability, perceived intelligence, and perceived safety of robots. International journal of social robotics, 1(1):71-81, 2009.

[23] A. Bangor, P. Kortum, and J. Miller. Determining what Individual SUS Scores Mean: Adding an Adjective Rating Scale. Journal of Usability Studies, 4(3):114-123, 2009. 